\title{
Effect of Prolonged Electrode Potential Cycling on the Conduction Properties of Poly(o-aminophenol) Film Electrodes
}

\author{
Ricardo I. Tucceri ${ }^{*}$ \\ Instituto de Investigaciones Fisicoquímicas Teóricas y Aplicadas (INIFTA), Sucursal 4, Casilla de Correo 16, 1900, La Plata Argentina \\ ${ }^{*}$ Corresponding author: rtucce@gmail.com
}

\begin{abstract}
The aim of this work was to study the effect of prolonged potentiodynamic cycling (PPC) on the conducting properties of poly(oaminophenol) (POAP) film electrodes. PPC reduces strongly the electron transport rate at POAP films. This effect is called herein deactivation. Cyclic Voltammetry (CV), Rotating Disc Electrode Voltammetry (RDEV) and Electrochemical Impedance Spectroscopy (EIS) were employed in this study. The attenuation of the voltammetric response of the polymer with the increase in the number of oxidation-reduction cycles allowed one to define a degree of deactivation. RDEV and EIS were employed to obtain the dependence of some charge-transport parameters on the degree of deactivation of the polymer. RDEV data were interpreted in terms of the electron hopping model. Impedance spectra were analysed on the basis of a model which considers a protonation reaction coupled with a self-exchange process between oxidized and reduced sites. POAP films maintain their conducting properties almost unaltered for about 500 potential cycles at a scan rate of $0.010 \mathrm{Vs}^{-1}$. However, a loss of conductivity was observed as the number of potential cycles was extended beyond 500 .
\end{abstract}

Keywords

Poly(o-aminophenol) Film Electrodes, Prolonged Potential Cycling (PPC), Deactivation, Charge-Transport Parameters

\section{Introduction}

The oxidation of o-aminophenol (o-AP) on different electrode materials (gold, platinum, carbon, indium-tin oxide, etc.) in aqueous medium is shown to form poly-o-aminophenol (POAP) [1-3]. $\mathrm{O}-\mathrm{AP}$ can be polymerized electrochemically in acidic, neutral and alkaline solutions. While a conducting film is only formed in acidic media, POAP synthesized in neutral and alkaline media leads to a nonconducting film [4]. The properties of POAP synthesized in basic medium are favorable to applications in the field of bioelectrochemistry and electrocatalysis [5]. The chargetransport process at POAP films synthesized in acid medium is mainly studied from the basic research viewpoint employing different electrochemical techniques $[1,3,6,7]$. POAP synthesized in acidic medium is also found to be a useful material to build electrochemical sensors and electrocatalysts. The development of nitric oxide [8] and ferric cations [9] sensors based on POAP synthesized in acid medium has been reported. POAP was also employed for the electrocatalytic detection of dihydronicotinamide adenine dinucleotides, $\mathrm{NAD}(\mathrm{P}) \mathrm{H}[10]$ and dissolved oxygen reduction [11]. Considering the interest in POAP synthesized in acid medium in both basic and applied research, not much attention has been paid to the decay of the electroactivity of POAP caused by its extensive use. The aim of the present work is to study how the charge-transport process at POAP films changes with PPC. It is expected that the present work will shed light on the limits of stability and durability of POAP, particularly when it is proposed as material to develop sensors and electrocatalysts.

\section{Experimental}

A gold rotating disc electrode (RDE) was used as base electrode to deposit POAP films. This gold RDE consisted of a gold rod press-fitted with epoxy resin into a Teflon sleeve so as to leave a $1 \mathrm{~cm}^{2}$ disc area exposed. The electrode was carefully polished with emery paper of decreasing grit size followed by alumina suspensions of $1,0.3$ and $0.05 \mu \mathrm{m}$, respectively, until a mirror-like finish was obtained. Then, it was submitted to ultrasonic cleaning to remove residual abraded polishing material. In order to obtain a more specular gold surface to deposit POAP films, a gold film about $50 \mathrm{~nm}$ in thickness was deposited by vacuum evaporation $[12-14]\left(\simeq 10^{-7}\right.$ Torr $)$ on the gold disc. The thickness of the evaporated gold thin film was determined as described in $[12,15]$. Then, in all experiments carried out in this work, POAP films were deposited on a specular gold film surface. POAP films were grown on these base electrodes following the procedure described in $[1,2]$. That is, polymer films were obtained by immersing the base electrode in a $10^{-3} \mathrm{M}$ orthoaminophenol $+0.4 \mathrm{M} \mathrm{NaClO}_{4}+$ $0.1 \mathrm{M} \mathrm{HClO}_{4}$ solution and cycling the potential between -0.25 and $0.8 \mathrm{~V}$ (versus SCE) at a scan rate $v=0.05 \mathrm{~V} \mathrm{~s}^{-1}$. In the same way as in [6,16-18], POAP films were grown up to an approximate thickness of $\phi_{p} \sim 60 \mathrm{~nm}$ by using a reduction charge $\left(Q_{R e d, T}=2.8\right.$ $\mathrm{mC} \mathrm{cm}{ }^{-2}$ ) versus the ellipsometric thickness working curve [16] These POAP-coated gold film electrodes were then rinsed and 
transferred to the supporting electrolyte solution $\left(0.4 \mathrm{M} \mathrm{NaClO}{ }_{4}\right.$ $+0.1 \mathrm{M} \mathrm{HClO}_{4}$ ) free of monomer, where they were stabilized by a continuous potential cycling at a scan rate of $0.01 \mathrm{Vs}^{-1}$. A typical voltammetric response of these films is shown in Fig. 1 (plot (a)). The POA nondeactivated films. A large-area gold grid was used as counterelectrode. All the potentials reported in this work are referred to the SCE.P films maintain this response on potential cycling within the potential range $-0.2 \mathrm{~V}<E<0.5 \mathrm{~V}$ up to 500 cycles. These POAP films are herein called nondeactivated films. A large-area gold grid was used as counterelectrode. All the potentials reported in this work are referred to the SCE.

A series of eight POAP-coated RDE was prepared (see first column in Table 1) and each one of them was successively employed in an individual experiment. That is, each POAP film was subjected to a different number of potential cycles (higher than 500 cycles, see second column in Table 1) within the potential region $-0.2 \mathrm{~V}<E<0.5 \mathrm{~V}$ in a deoxygenated supporting electrolyte solution. Then, the corresponding $\mathrm{j}$-E responses for each one of the eight POAP films were recorded. An attenuation of the voltammetric response was observed for these films when the number of potential cycles was higher than 500 (Fig. 1). These POAP films are herein called deactivated films. Then, RDEV experiments were performed with both nondeactivated and deactivated POAP films in the presence of a solution containing equimolar concentrations of p-benzoquinone $(\mathrm{Q})$ and hydroquinone $(\mathrm{HQ})$ species $\left(0.1 \mathrm{M} \mathrm{HClO}_{4}+0.4 \mathrm{M} \mathrm{NaClO}_{4}+2 \times 10^{-3} \mathrm{M} \mathrm{Q} / \mathrm{HQ}\right)$. Stationary current-potential curves (I-E) at different electrode rotation rates, $\Omega$, were recorded. From these curves, cathodic and anodic limiting current versus electrode rotation rate $\left(I_{\text {lim }}\right.$ versus $\left.\Omega^{1 / 2}\right)$ dependences were obtained. In some RDEV experiments the HQ/Q redox couple concentration in solution was varied. Ac impedance diagrams at potential values $\mathrm{E}<0.0 \mathrm{~V}$ (SCE) were also recorded for nondeactivated and deactivated POAP films in the presence of the supporting electrolyte solution $\left(0.4 \mathrm{M} \mathrm{NaClO}_{4}\right.$ $+0.1 \mathrm{M} \mathrm{HClO}_{4}$ ).

In $\mathrm{CV}$ and RDEV measurements a PAR Model 173 potentiostat and a PAR Model 175 function generator were used. An X1-X2-Y Hewlett-Packard Model 7046 B plotter was used to record $j-E$ and steady-state current-potential curves $I-E$. The electrode rotation speed, $\Omega$, was controlled with homemade equipment that allows one to select a constant $\Omega$ in the range $50 \mathrm{rev}$ $\min ^{-1}<\Omega<7000 \mathrm{rev} \mathrm{min}^{-1}$. This was periodically controlled with a digital photo tachometer (Power Instruments Inc., model 891). Impedance spectra were measured following a 30-min application of the steady-state potential ranging from $-0.35 \mathrm{~V}$ to $0.0 \mathrm{~V}$. Impedance values were determined at seven discrete frequencies per decade with a signal amplitude of $5 \mathrm{mV}$. The validation of the impedance spectra was done by using Kramers-Kronig transformations. Impedance measurements in the frequency range 0.01 $\mathrm{Hz}-10 \mathrm{kHz}$ were performed with a PAR 309 system.

AR grade chemicals were used throughout. O-aminophenol (Fluka) was purified as described elsewhere $[1,2] . \mathrm{HClO}_{4}$ and $\mathrm{NaClO}_{4}$ (Merck) were used without further purification. Benzoquinone and hydroquinone (Merck) were also used without purification. The solutions were prepared with water purified using a Millipore Milli-Q system.

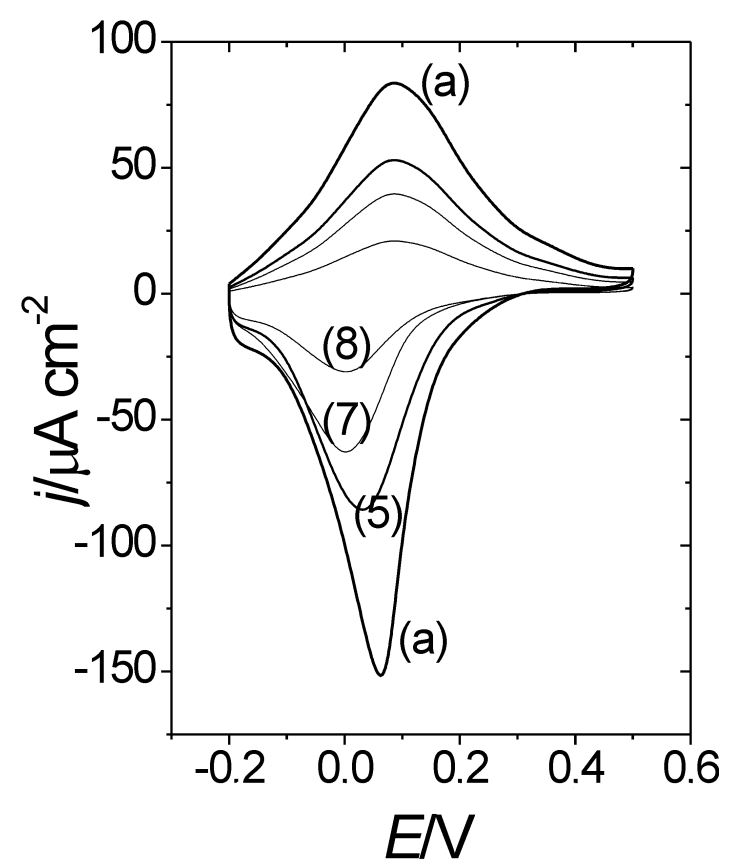

Figure 1. Voltammetric $(j-E)$ responses for a $2.8 \mathrm{mC} \mathrm{cm}^{-2}\left(\phi_{p}=60\right.$ $\mathrm{nm})$ thick POAP film. (a) A nondeactivated POAP film $\left(\theta_{c}{ }^{d}=0\right)$. Curves (5), (7) and (8) correspond to films whose degree of deactivation is: $\theta_{c}{ }^{d}=0.42 ; \theta_{c}{ }^{d}=0.62$ and $\theta_{c}{ }^{d}=0.74$, respectively. Electrolyte: $0.1 \mathrm{M} \mathrm{HClO}_{4}+0.4 \mathrm{M} \mathrm{NaClO}_{4}$. Scan rate: $v=0.01 \mathrm{~V} \mathrm{~s}^{-1}$.

\section{Results and Discussion}

\subsection{Voltammetric responses of nondeactivated and de- activated POAP films}

The voltammetric response corresponding to a nondeactivated POAP film within the potential range comprised between -0.2 $\mathrm{V}$ and $0.5 \mathrm{~V}$ is shown in Fig. 1 (plot (a)). As was indicated, the POAP film maintains this response with potential cycling within the potential range $-0.2 \mathrm{~V}<E<0.5 \mathrm{~V}$ up to 500 potential cycles (scan rate $0.01 \mathrm{Vs}^{-1}$ ). However, after a higher number of potential cycles this response start to change. Figure 1 compares the $j-E$ responses of a nondeactivated POAP film (plots (a)) with those of the films (5), (7) and (8) (see first column of Table 1) that were subjected to the corresponding number of potential cycles $(>500)$ indicated in column 2 of Table 1 . The more attenuated voltammetric response observed in Fig. 1, as the number of potential cycles increases indicates a deactivation of the POAP film. In this regard, voltammetric reduction charge values corresponding to the completely reduced POAP films were compared for a nondeactivated film $\left(Q_{\text {Red }, T}=2.8 \mathrm{mC} \mathrm{cm}^{-2}\right)$ and the different deactivated films $\left(\mathrm{Q}_{\text {Red,c }}\right)$ indicated in Table 1 . Then, a degree of deactivation (column 4 of Table 1) was defined as

$$
\theta_{c}{ }^{d}=1-\left(Q_{\text {Red }, c} / Q_{\text {Red }, T}\right)
$$

$Q_{R e d, c}$ is the total reduction charge assessed by integration of the corresponding $j-E$ response from $\mathrm{E}=0.5 \mathrm{~V}$ towards the 
Table 1. Effect of the Number of Potential Cycles on the Voltammetric Charge of a POAP Film

\begin{tabular}{|l|l|l|l|}
\hline $\begin{array}{l}\text { aPOAP } \\
\text { film }\end{array}$ & $\begin{array}{l}{ }^{\mathrm{b}} \text { Number } \\
\text { of Potential } \\
\text { cycles }\end{array}$ & ${ }^{\mathrm{c}} Q_{\text {Red }, c} / \mathrm{mC} \mathrm{cm}^{-2}$ & ${ }^{\mathrm{d}} \boldsymbol{\theta}_{c}{ }^{\mathrm{d}}$ \\
\hline 1 & 624 & 2.58 & 0.08 \\
\hline 2 & 1248 & 2.30 & 0.18 \\
\hline 3 & 1872 & 2.10 & 0.25 \\
\hline 4 & 2496 & 1.85 & 0.34 \\
\hline 5 & 3120 & 1.62 & 0.42 \\
\hline 6 & 3744 & 1.37 & 0.51 \\
\hline 7 & 4368 & 1.06 & 0.62 \\
\hline 8 & 4992 & 0.73 & 0.74 \\
\hline
\end{tabular}

a Numbers 1 to 8 represent different deactivated POAP films.

b Number of potential cycles to which each POAP film was subjected in the supporting electrolyte solution (scan rate: $0.01 \mathrm{~V}$ $\left.\mathrm{s}^{-1}\right)$.

c Voltammetric reduction charge of the different deactivated POAP films after being subjected to the number of potential cycles indicated in column 2.

${ }^{d}$ Degree of deactivation of each one of the POAP films after being subjected to the number of potential cycles indicated in column 2. The degree of deactivation was calculated from $\theta_{c}{ }^{d}=$ $1-\left(Q_{\text {Red }, c} / Q_{\text {Red }, T}\right)$, where $Q_{\text {Red }, T}\left(=2.8 \mathrm{mC} \mathrm{cm}^{-2}\right)$ is the voltammetric reduction charge of a nondeactivated film.

negative potential direction for a deactivated film, and $Q_{\text {Red }, T}=$ $2.8 \mathrm{mC} \mathrm{cm}^{-2}$ is the total reduction charge for the nondeactivated film. In this way, for a nondeactivated POAP film (plot (a) in Fig. 1) the degree of deactivation was $\theta_{c}{ }^{d}=0$, taking $Q_{\text {Red }, T}=2.8$ $\mathrm{mC} \mathrm{cm}^{-2}$ as reference charge. However, values of $\theta_{c}{ }^{d}>0$ are indicative of POAP films that have been deactivated.

\subsection{Rotating Disc Electrode Voltammetry in the pres- ence of a hydroquinone/p-benzoquinone solution}

As was indicated, RDEV was employed to study the behaviour of nondeactivated and deactivated POAP films in contact with an electroactive solution containing the hydroquinone/p-benzoquinone redox couple. In the presence of a redox active solution, besides charge transfer between redox sites of the polymer and the external redox couple, the oxidation and reduction of fixed sites introduce charged sites into the polymer film, which, in order to achieve charge neutrality, require the ingress of counterions from the contacting electrolyte solution and, according to the Donnan relation, the egress of co-ions. Disregarding the chargetransport control, a redox polymer undergoing electrolysis may follow Fick's diffusion law and usually Fick's formalism agrees with the experimental results. Electron hopping is believed to be the mechanism for electron transport at polymer materials, but it is also possible that ion motions may partially or totally control the rate of charge transport. Then, steady-state current-potential curves obtained in this work were interpreted on the basis of the traditional electron hopping model [17-19].

In previous work [20] RDEV experiments at gold electrodes coated with POAP films were carried out to study the diffusion

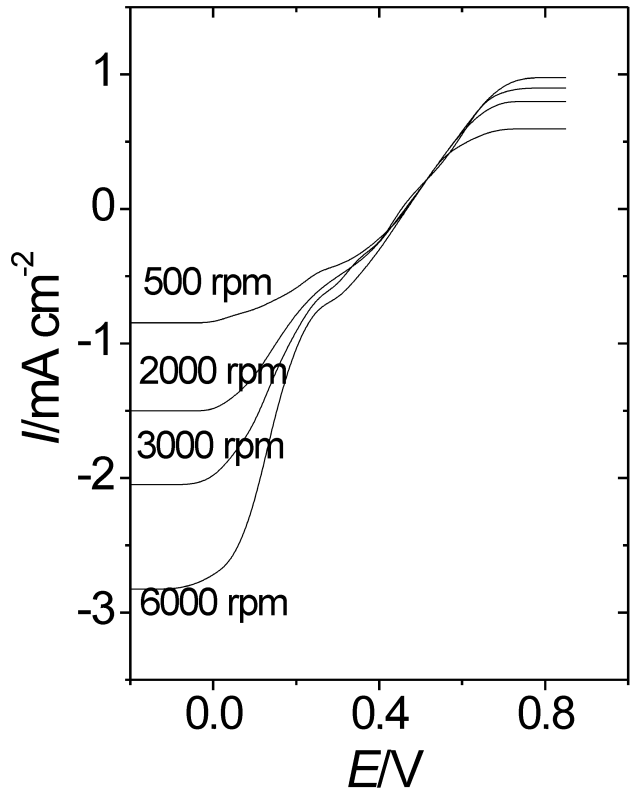

Figure 2. Steady-state current-potential $(I-E)$ curves for different rotation rates $(\Omega)$ of the rotating disc electrode. A nondeactivated POAP film. $\Omega$ Values are indicated in the figure. Film thickness: $60 \mathrm{~nm}$. Electrolyte: $0.1 \mathrm{M} \mathrm{HClO}_{4}+0.4 \mathrm{M} \mathrm{NaClO}_{4}+2 \times 10^{-3} \mathrm{M}(\mathrm{HQ} / \mathrm{Q})$.

processes of benzoquinone (Q) and hydroquinone (HQ) species through nondeactivated films. Diffusion-limited currents at $\mathrm{E}<$ $0.0 \mathrm{~V}$ for $\mathrm{Q}$ reduction and at $\mathrm{E}>0.8 \mathrm{~V}$ for $\mathrm{HQ}$ oxidation were observed. While the anodic limiting current corresponds to the oxidation of HQ species that penetrate through the polymer film to reach the metal surface, cathodic limiting currents for $\mathrm{Q}$ reduction are related to a rapid electron-transfer mediation at the POAP_redox active solution interface, which occurs without significant penetration of $\mathrm{Q}$ into the polymer layer. As we are interested in the electron-transport process across POAP films, only the electrochemical behavior of nondeactivated and deactivated POAP films at negative potential values $(\mathrm{E}<0.0 \mathrm{~V})$ was considered in this work.

Figure 2 shows stationary current-potential curves $(I-E)$ at different electrode rotation rates, $\Omega$, for a nondeactivated POAP film contacting a $0.1 \mathrm{M} \mathrm{HClO}_{4}+0.4 \mathrm{M} \mathrm{NaClO} \mathrm{Na}_{4}+2 \times 10^{-3} \mathrm{M}$ $\mathrm{Q} / \mathrm{HQ}$ solution. (I-E) curves at different $\Omega$ values were also obtained for each one of the deactivated POAP films indicated in Table 1. For instance, Fig. 3 shows this representation for a POAP film with $\theta_{c d}=0.51$. As can be seen by comparing Figs. 2 and 3 , at each electrode rotation rate, both anodic and cathodic limiting currents for a deactivated POAP film are lower than those for a nondeactivated one. Also, after a given electrode rotation rate, which depends on the degree of deactivation, the cathodic limiting current for a deactivated film becomes independent of this variable. $I_{\text {lim }, c}$ versus $\Omega^{1 / 2}$ dependences at potential values $\mathrm{E}$ $<0.0 \mathrm{~V}$ for both nondeactivated and deactivated POAP films are 
shown in Fig. 4.

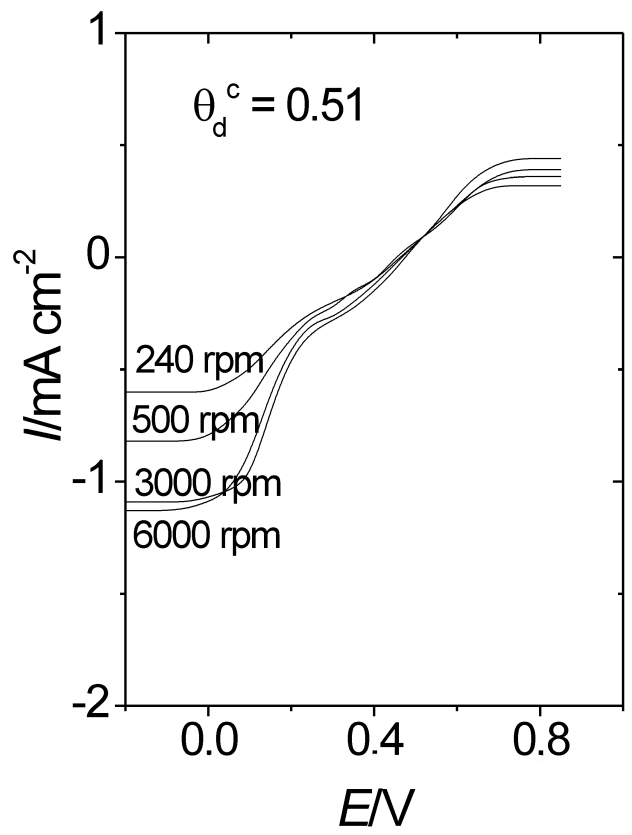

Figure 3. Steady-state current-potential $(I-E)$ curves for different rotation rates $(\Omega)$ of the rotating disc electrode. A deactivated POAP film $\left(\theta_{c}{ }^{d}=0.51\right) . \Omega$ Values are indicated in the figure. Film thickness: $60 \mathrm{~nm}$. Electrolyte: $0.1 \mathrm{M} \mathrm{HClO}_{4}+0.4 \mathrm{M} \mathrm{NaClO}_{4}+2 \times 10^{-3} \mathrm{M}(\mathrm{HQ} / \mathrm{Q})$.

For a nondeactivated POAP film a linear $I_{\text {lim,c }}$ versus $\Omega^{1 / 2}$ dependence, which follows the Levich equation, is obtained within a wide range of $\Omega$ values. However, for POAP films that have been deactivated, after a certain $\Omega$ value, a constant cathodic limiting

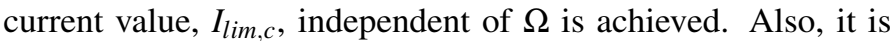
observed that the transition at which the cathodic limiting current becomes independent of $\Omega$ occurs at lower $\Omega$ values as the degree of deactivation increases. The explanation of this effect can be given in terms of the electron hopping model [17-19]. Limiting current values at which $I_{l i m, c}\left(=I_{e}\right)$ becomes constant were considered as a representation of the maximum flux of electrons confined in the polymer, according to Eq. (2) [19]:

$$
I_{e}=n F A D_{e} c / \phi_{p}
$$

In Eq. (2), $c$ is the concentration of redox sites of the polymer and $\phi_{p}$ the polymer film thickness. $D_{e}$ represents a measure of the electron hopping rate and $n$ expresses the numbers (fractions) of unit charges per monomer unit of the polymer. $A$ is the electrode area and $\mathrm{F}$ the Faraday's constant. Experimental $I_{e}$ values, corresponding to each one of the eight deactivated POAP films contacting a $2 \times 10^{-3} \mathrm{M} \mathrm{HQ} / \mathrm{Q}$ solution, were extracted from the cathodic plateau and they are listed as a function of $\theta_{c}{ }^{d}$ in Table 2.

As can be seen from Table 2, $I_{e}$ decreases with increasing $\theta_{c}{ }^{d}$. In this regard, such a constant value of the current $\left(I_{e}\right)$ at a given $\Omega$ value for deactivated POAP films can be related to a slow electron

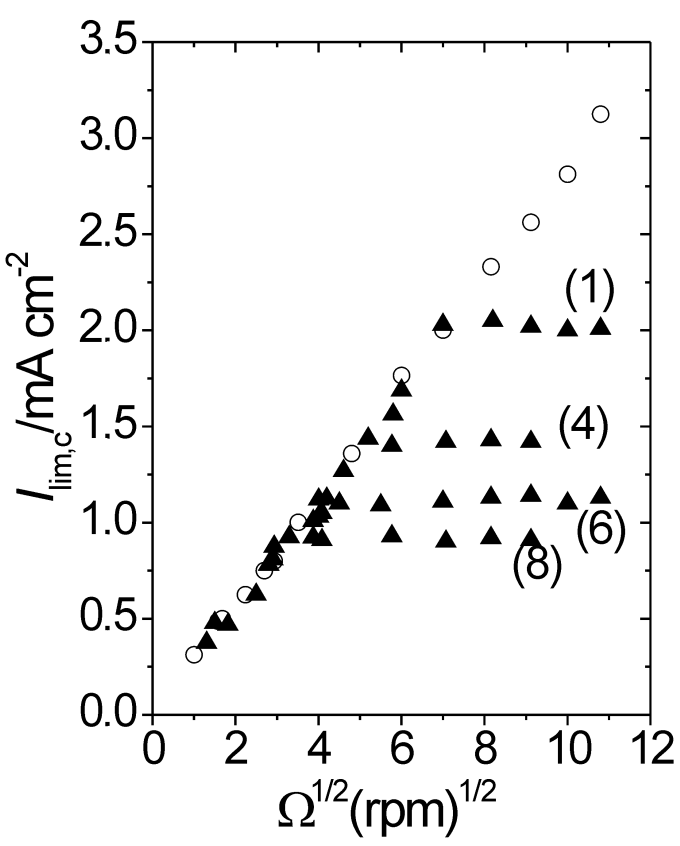

Figure 4. Levich representations $I_{\text {lim,c }}$ versus $\Omega^{1 / 2}$ for POAP films contacting a $0.1 \mathrm{M} \mathrm{HClO}_{4}+0.4 \mathrm{M} \mathrm{NaClO}_{4}+2 \times 10^{-3} \mathrm{M}(\mathrm{HQ} / \mathrm{Q})$ solution. (O) A nondeactivated POAP film. (1), (4), (6) and (8) correspond to the four films indicated in Table 1.

transport across the POAP film to mediate in the electron-transfer reaction at the polymer-solution interface, as compared with a nondeactivated POAP film. As one increases the flux of Q (increase of $\Omega$ ) from the bulk solution, then if the flux exceeds the supply of electrons from the electrode through the polymer to the electrolyte interface, the rate-limiting step will shift from the limiting transport of $\mathrm{Q}$ to the limiting transport of the charge through the polymer. In order to verify this limiting chargetransport process across the POAP film, the HQ/Q concentration in solution was varied. It is found that the constant current for a given deactivated film remains unchanged with changing redox couple concentration in solution. According to Eq. (2), the slower electron transport in deactivated films, as compared with nondeactivated ones, could be attributed to a decrease of De. By employing the Ie values shown in Table 2 and using the parameter values $c=4.7 \mathrm{M}$ [1], $A=1 \mathrm{~cm}^{2}, n=0.44$ [21] and $\phi_{p}=60 \mathrm{~nm}$ in Eq. (2), one obtains a decrease of $D_{e}$ from $6.01 \times 10^{-11}$ to $2.76 \times 10^{-11} \mathrm{~cm}^{2} \mathrm{~s}^{-1}$ for a relative increase of $\theta_{c}$ from 0.08 to 0.74 .

The electron diffusion coefficient, $D_{e}$, in electroactive materials has been expressed in terms of the mean distance between adjacent active redox sites [22], according to $D_{e}=\left(a^{2} k_{0}\right)$, where $k_{0}$ is the intermolecular electron-transfer rate constant and a is the mean distance between two adjacent redox sites. The hopping rate, $k_{0}$, exhibits an exponential dependence on a, through the energy $-U(x+a)$ of a state with an electron in the position $x$ along the current direction (see eq. (23) in Ref. [22]). In this 
Table 2. Electron Current $I_{e}$ as a Function of the Degree of Deactivation $\theta_{c}{ }^{d}$ of a POAP Film

\begin{tabular}{ccc}
\hline${ }^{\mathrm{a}}$ POAP film & ${ }^{\mathrm{b}} \theta_{c}{ }^{d}$ & ${ }^{\mathrm{c}} I_{e} / \mathrm{mAcm}^{-2}$ \\
\hline 1 & 0.08 & 2.02 \\
\hline 2 & 0.18 & 1.64 \\
\hline 3 & 0.25 & 1.46 \\
\hline 4 & 0.34 & 1.40 \\
\hline 5 & 0.42 & 1.21 \\
\hline 6 & 0.51 & 1.12 \\
\hline 7 & 0.62 & 0.98 \\
\hline 8 & 0.74 & 0.92
\end{tabular}

a Numbers 1 to 8 represent different deactivated POAP films.

${ }^{b}$ Degree of deactivation of each one of the POAP films after being subjected to the potential number of cycles indicated in column 2 of Table 1.

c Electron current $I_{e}$ as a function of the degree of deactivation, $\theta_{c}{ }^{d}$ obtained by RDEV in the presence of the HQ/Q redox couple.

respect, a $k_{0}$ decrease should be expected as the hopping distance a increases. The decrease of $D_{e}$ obtained from Eq. (2) could be attributed to an increase of the hopping distance between remnant active redox sites after deactivation.

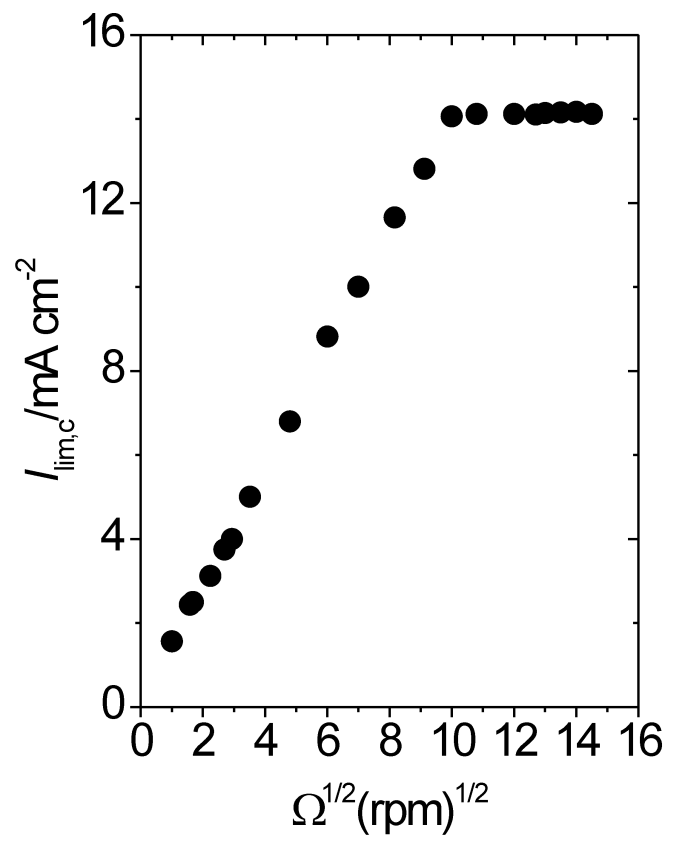

Figure 5. Levich representation $I_{\text {lim }, c}$ versus $\Omega^{1 / 2}$ for a nondeactivated POAP film contacting a $0.1 \mathrm{M} \mathrm{HClO}_{4}+0.4 \mathrm{M} \mathrm{NaClO}_{4}+0.01 \mathrm{M}(\mathrm{HQ} / \mathrm{Q})$ solution.

It is interesting to remark that a constant current Ie independent of $\Omega$ is also obtained for a nondeactivated POAP film in contact with a $0.1 \mathrm{M} \mathrm{HClO}_{4}+0.4 \mathrm{M} \mathrm{NaClO}_{4}+2 \times 10^{-3} \mathrm{M} \mathrm{Q} / \mathrm{HQ}$ solution. However, in this case the constant current is obtained at very high
Table 3. EFICIENCY $\left(I_{e} / I_{o} \times 100\right)$ OF POAP TO ACT AS MEDIATOR IN THE HQ/Q REDOX REACTION

\begin{tabular}{cccc}
\hline POAP Film & $\theta_{c}{ }^{d}$ & $I_{e} / \mathrm{mAcm}^{-2}$ & $I_{e} / I_{o} \times 100$ \\
\hline 1 & 0,08 & 2.02 & 90 \\
\hline 2 & 0,18 & 1.64 & 74 \\
\hline 3 & 0,25 & 1.46 & 66 \\
\hline 4 & 0,34 & 1.40 & 64 \\
\hline 5 & 0,42 & 1.21 & 58 \\
\hline 6 & 0,51 & 1.12 & 51 \\
\hline 7 & 0,62 & 0.98 & 44.5 \\
\hline 8 & 0,74 & 0.92 & 42 \\
\hline
\end{tabular}

electrode rotation rates $(\Omega>8000 \mathrm{rpm})$. It is possible that at high angular speeds of the rotating disc electrode, the flux into the bulk solution would not longer laminar, so that the proportionality between the current and $\Omega^{1 / 2}$, should not be expected. However, if the HQ/Q concentration is increased $(0.01 \mathrm{M})$ a constant current Ie $\left(\approx 15 \mathrm{~mA} \mathrm{~cm}^{-2}\right)$ is observed for a nondeactivated film at about $7000 \mathrm{rpm}$ (Fig. 5). As this value is reproducible and it decreases as the deactivation of the polymer increases, then our interpretation of a change in the rate limiting process during the mediated electron-transfer reaction seems to be correct. Similar effects were observed for other polymers [23]. Considering the constant current value obtained for high $\mathrm{HQ} / \mathrm{Q}$ concentrations, a $D_{e}$ about $1.22 \times 10^{-10} \mathrm{~cm}^{2} \mathrm{~s}^{-1}$ is obtained for a nondeactivated POAP film. This finding seems to indicate that a restriction for the electron transport also occurs across a nondeactivated POAP film in contact of a concentrated electroactive solution and high enough flux into the bulk solution.

On the basis of data showed in Table 2 and Fig. 4, one can assess the efficiency of POAP to act as mediator in the HQ/Q redox reaction. By taking as reference a high enough electrode rotation rate $(\Omega=3800 \mathrm{rpm})$ to reach a constant current for all the deactivated films treated in the work (see Fig. 4), one can calculate the $\left(I_{e} / I_{o} \times 100\right)$ ratio, where $I_{e}$ are the different constant current values for the different deactivated film (see Table 2$)$ and $I_{o}$ is the current value $\left(2.2 \mathrm{~mA} \mathrm{~cm}^{-2}\right)$ corresponding to the nondeactiavated film, which follows the Levich relationship at $\Omega=3800 \mathrm{rpm}$ (see Fig. 4). Then, one can build the following Table 3 .

In Table 3, $\left(I_{e} / I_{o} \times 100\right)$ represents the efficiency of POAP to act as mediator in the HQ/Q redox reaction. For a nondeactivated film, $I_{o}=I_{e}=2.2 \mathrm{~mA} \mathrm{~cm}^{-2}$, then, $I_{e} / I_{o}=1$ and $\left(I_{e} / I_{o} \times 100\right)=$ 100, which corresponds to the maximal efficiency. As can be seen from the Table 3, the efficiency of POAP deceases with the PPC from 100 to about 40 per cent, as the number of potential cycles increases from around 500 to 5000 .

\subsection{Ac impedance measurements and interpretation of impedance spectra}

The aim of this part of the work is to study the charge conduction process at POAP films employing ac impedance measurements and then, compare charge-transport parameters obtained from both RDEV and EIS techniques. As the electroactivity of POAP is explained by a redox mechanism that involves an ad- 
dition/elimination of protons coupled with a reversible electron transfer [6], a modified electron hopping model, which considers a protonation reaction, developed by us in [24] was used to obtain charge-transport parameters of nondeactivated and deactivated POAP films in the sole presence of the supporting electrolyte solution. In this model, a protonation reaction (Reaction (I) see below) is coupled to a self-exchange process between oxidized and reduced sites (Reaction II, see below)

$$
\begin{aligned}
& O+\left[H^{+}\right]_{f i l m} \underset{k_{\prime}^{1}}{\stackrel{k_{\prime}^{-1}}{\longrightarrow}} H O^{+}(\mathrm{I}) \\
& \mathrm{OH}^{+}+e^{-} \underset{k_{f}}{\stackrel{k_{b}}{\longrightarrow}} \mathrm{RH} \text { (II) }
\end{aligned}
$$

The analytical expression for the impedance derived in [27] is:

$$
Z(\omega)=R_{Q t}+\left(R T / n F^{2} A c\right)\left[k_{f}+k_{b}(K+1) / K\right] A\left(\omega, D_{e}, k\right)
$$

$R_{Q t}$ in Eq. (3) is a charge-transfer resistance, which is given by the expression:

$$
R_{Q t}=\left(R T / n F^{2} A c\right)\left[\left(k_{f}+k_{b}\right)\left(k_{f} k_{b}\right)^{-1}+1 / K k_{f}\right]
$$

In Eqs. (3) and (4), $A$ is the electrode area, $c$ the volumetric redox site concentration, and $K$ an equilibrium constant that can be explicitly written in terms of the solution $\mathrm{pH}$ as :

$$
K=\left(k_{1}^{\prime} / k_{-1}^{\prime}\right) K_{p} 10^{-p H}
$$

$K$ also depends on the $k_{1}^{\prime}$ and $k_{-1}^{\prime}$ constants involved in step (I) and a partition coefficient $K_{p}$, which determines the ratio between the proton concentration inside the film $\left(\left[\mathrm{H}^{+}\right]_{\text {film }}\right)$ and the actual proton concentration in solution $\left(\left[\mathrm{H}^{+}\right]_{\text {sol }}\right)$, i.e.:

$$
\left[H^{+}\right]_{\text {film }}=K_{p}\left[H^{+}\right]_{\text {sol }}
$$

$k_{f}$ and $k_{b}$ in Eq. (3) are the forward and backward electrochemical rate constants involved in step (II), respectively, and $A\left(\omega, D_{e}, k\right)$ is a function of the frequency, $\omega$, which also contains an affective diffusion coefficient $\left(D_{e}\right)$ to describe the charge-transport process within the polymer film and the constant $k=k_{-1}^{\prime}+\left[H^{+}\right]_{\text {film }} k_{1}^{\prime}$ (see eq. (33) in Ref. [24]). $\mathrm{OH}^{+}$and RH (steps I and II) are the protonated oxidized and reduced forms of the polymer confined redox couple, respectively. Other chemical equilibria following the self-exchange (II) were ignored in [24]. Concerning step (II), the electroactive centres $\mathrm{OH}+$ and $\mathrm{RH}$ can exchange electrons with the electrode at the metal/polymer interface following a Buttler-Volmer kinetics, with $k_{f}$ and $k_{b}$ given by the expressions:

$$
\begin{aligned}
& k_{f}=k^{s h} \exp \left[b_{f}\left(E-E^{0}\right)\right] \\
& k_{b}=k^{s h} \exp \left[-b_{b}\left(E-E^{0}\right)\right]
\end{aligned}
$$

$E^{0}$ in expressions (7) and (8) is the standard potential of the redox couple, $b_{f}$ and $b_{b}$ are the Tafel coefficients, $b_{f}=\alpha n F / R T$ and $b_{b}=(1-\alpha) n F / R T$, and $k_{s h}$ is the electrochemical standard rate constant. The other constants have their usual meanings.

The redox centres are uniformly distributed throughout the polymer with a total concentration, $c$, given by:

$$
c=[O]+\left[H O^{+}\right]+[R H]
$$

Eq. (3) was employed to fit experimental impedance diagrams of nondeactivated and deactivated POAP films in the presence of the supporting electrolyte. Deactivation by prolonged potential cycling was considered to only affect the charge-transfer process at the metal/polymer interface $\left(R_{Q t}\right)$ and the charge- diffusion process $\left(D_{e}\right)$ within the polymer film. As the solution $\mathrm{pH}$ and electrolyte composition remain constant, the other parameters of the model were assumed to be independent of the deactivation process, and values for nondeactivated POAP films obtained in [24] were employed.

Nyquist diagrams at the reduced state $(E=-0.2 \mathrm{~V})$ for a nondeactivated POAP film and deactivated films (5), (7) and (8) indicated in Table 1 , respectively, in contact with a $0.1 \mathrm{M} \mathrm{HClO}_{4}$ $+0.4 \mathrm{M} \mathrm{NaClO}_{4}$ solution, are compared in Fig. 6 (see discrete points).

The early increase in the imaginary component of the impedance and the absence of any semicircle at high frequency for a nondeactivated film are indicative of a fast interfacial charge-transfer process (plot (a) in Fig. 6). However, a well-defined high-frequency semicircle is observed in the impedance diagrams of POAP films subjected to extensive potential cycling. As the number of potential cycles increases, the high-frequency semicircle is more pronounced. The increase in the size of the high-frequency semicircle in Nyquist plots is indicative of a restriction in the chargepropagation process across the gold/POAP/solution system.

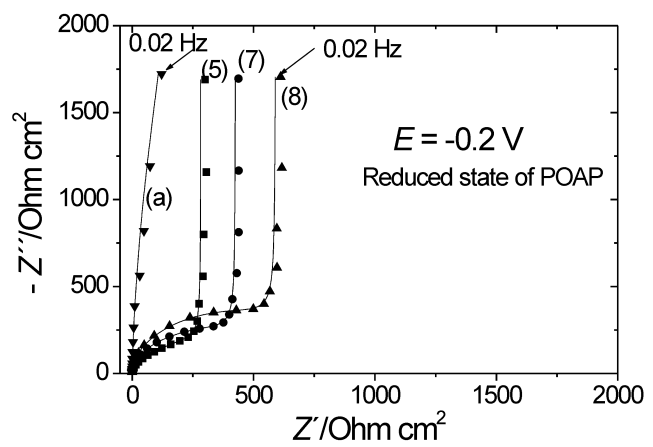

Figure 6. Nyquist diagrams for (a) a nondeactivated POAP film and for the deactivated films (5), (7) and (8) indicated in Table 1. $E=-0.2 \mathrm{~V}$. Discrete points are experimental data and continuous lines represent the fitting by using de model described in [24]. Electrolyte: $0.1 \mathrm{M} \mathrm{HClO}_{4}+$ $0.4 \mathrm{M} \mathrm{NaClO}_{4}$. Thickness of the POAP film: $60 \mathrm{~nm}$.

Continuous lines on the Nyquist plots shown in Fig. 6 are simulated curves calculated by using Eq. (3). A good fitting was observed for all impedance diagrams. In the same way as in the previous model, the fitting procedure by using Eq. (3) was based on the CNLS (Complex Nonlinear Squares) method. A rigorous fitting procedure was performed where the error structure was assessed following the references [25-28]. Continuous lines in Fig. 6 represent the weighted complex nonlinear least-squares fit to the data. Real and imaginary parts versus frequency plots corresponding to each one of the Nyquist representations were also built. One of these representations is shown in Fig. 7.

In the simulations the following parameter values were considered as known and constants: number of transferred electrons, 
$n(=0.44[21]) ;$ polymer thickness, $\phi_{p}(=60 \mathrm{~nm}[16,17])$; total redox site concentration, $c\left(=4.7 \times 10^{-3} \mathrm{~mol} \mathrm{~cm}^{-3}[1]\right) ; E^{0}(=$ $0.015 \mathrm{~V}$ versus SCE [21]); $K(=0.085$ for $p H 1$ [24]) and $k(=0.18$ $\mathrm{s}^{-1}$ ) [24]. Then, the remnant parameters contained in Eq. (3), i.e., $k_{s h}$ and $D_{e}$, were calculated from experimental impedance data by the fitting procedure described above. Although the parameter $k_{s h}$ varied without restraints during the fitting, for POAP film thickness $\left(\phi_{p}=60 \mathrm{~nm}\right)$ and solution $p H=1$, used in this work, $D_{e}$ was allowed to vary within the range $10^{-7}-10^{-11} \mathrm{~cm}^{2} \mathrm{~s}^{-1}$. De values lower than $10^{-11} \mathrm{~cm}^{2} \mathrm{~s}^{-1}$ were considered unrealistic for thick POAP films. A contribution of the interfacial capacitance, $C_{H}$, considered as a fitting parameter, was included in order to represent the actual impedance diagrams from the calculated ones.

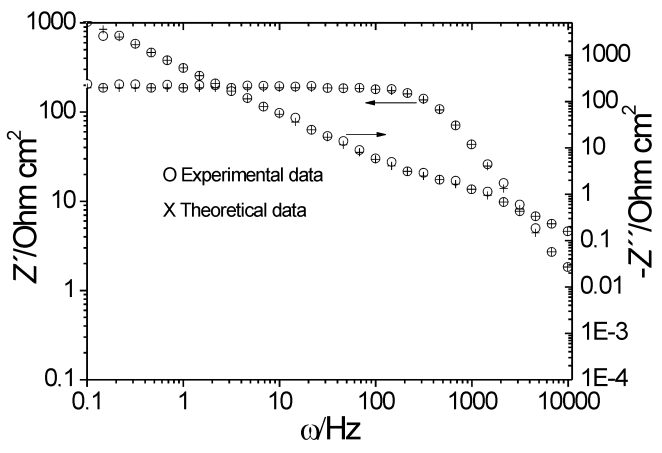

Figure 7. Real part $\left(Z^{\prime}\right)$ and imaginary part $\left(-Z^{\prime \prime}\right)$ versus frequency $(\omega)$ plots (point to point representations) for a nondeactivated POAP film. $E=-0.2 \mathrm{~V}$. Electrolyte: $0.1 \mathrm{M} \mathrm{HClO}_{4}+0.4 \mathrm{M} \mathrm{NaClO}_{4}$. POAP film thickness: $60 \mathrm{~nm}$. (O) Experimental data; (+) the fitting employing Eq. (3).

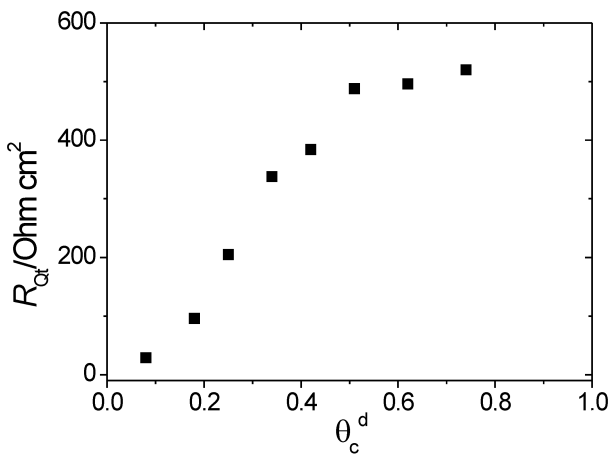

Figure 8. $R_{Q t}$ versus $\theta_{c}{ }^{d}$ dependence extracted employing the model described in [24].

Dependences of the electrochemical standard rate constant $\left(k_{s h}\right)$ and the electron diffusion coefficient $\left(D_{e}\right)$ on the degree of deactivation $\left(\theta_{c}{ }^{d}\right)$ were extracted from the fitting procedure. Values of the fitting parameters, $k_{s h}$ and $D_{e}$, for the different deactivated POAP films are listed in Table 4. As can be seen from Table 4 , both $k_{s h}$ and $D_{e}$ decrease as the POAP film becomes more deactivated with prolonged potential cycling. Similar $k_{s h}$ values
Table 4. STANDARD ELECTROCHEMICAL RATE CONSTANT AND DIFFUSION COEFFICIENT VALUES FOR DEACTIVATED POAP FILMS EXTRACTED FROM THE IMPEDANCE MODEL GIVEN IN [24]

\begin{tabular}{cccc}
\hline${ }^{\mathrm{a}}$ POAP Film & ${ }^{\mathrm{a}} 10^{5} k_{s h} / \mathrm{cm} \mathrm{s}^{-1}$ & ${ }^{\mathrm{c}} 10^{10} D_{e} / \mathrm{cm}^{2} s^{-1}$ & ${ }^{\mathrm{d}} \theta_{c}{ }^{d}$ \\
\hline 1 & 13.6 & 2.23 & 0.08 \\
\hline 2 & 4.11 & 1.64 & 0.18 \\
\hline 3 & 3.14 & 1.35 & 0.25 \\
\hline 4 & 2.16 & 0.84 & 0.34 \\
\hline 5 & 2.03 & 0.54 & 0.42 \\
\hline 6 & 1.63 & 0.41 & 0.51 \\
\hline 7 & 0.81 & 0.34 & 0.62 \\
\hline 8 & 0.77 & 0.29 & 0.74
\end{tabular}

${ }^{\mathrm{a}}$ Numbers 1 to 8 represent different deactivated POAP films.

${ }^{b}$ Values of the standard electrochemical rate constant, ksh, for the different degrees of deactivation given in column 4 . The value of ksh for a nondeactivated film extracted from the fitting using Eq. (3) is $2.7 \times 10^{5} \mathrm{~cm} \mathrm{~s}^{-1}$.

${ }^{c}$ Diffusion coefficient values for the different degrees of deactivation given in column 4 . The De value for a nondeactivated film (subjected to a number of potential cycles lower than 500) extracted from the fitting using Eq. (3) is $2.47 \times 10^{-9} \mathrm{~cm}^{2} \mathrm{~s}^{-1}$.

${ }^{d}$ Degree of deactivation of each one of the POAP films after being subjected to the number of potential cycles indicated in column 2 of Table 1 . The degree of deactivation was calculated from $\theta_{c}{ }^{d}=1-\left(Q_{\text {Red }, c} / Q_{\text {Red,T }}\right)$, where $Q_{\text {Red }, T}\left(=2.8 \mathrm{mC} \mathrm{cm}^{-2}\right)$ is the voltammetric reduction charge of a nondeactivated film.

for deactivated POAP films shown in Table 4 were previously reported for POAP in [21]. Low values of $k_{s h}$ indicate partial deactivation of the polymer. However, high $k_{s h}$ values $\left(2.7 \times 10^{5}\right.$ $\mathrm{cm} \mathrm{s}^{-1}$ ) such as that obtained in this work from impedance diagrams (see notes at the foot of Table 4), which do not show an appreciable high-frequency semicircle (plot (a) in Fig. 6), should correspond to a polymer matrix relatively free of deactivated zones. The $R_{Q t}$ versus $\theta_{c}{ }^{d}$ dependence, extracted from $k_{s h}$ values given in column 2 of Table 4, was represented in Fig. 8.

As was indicated, although electron hopping is believed to be the mechanism for electron transport at polymer materials, it is also possible that ion motions may partially or totally control the rate of charge transport. Then, $D_{e}$ obtained with the model derived in [24] can be considered as an effective or binary diffusion coefficient value. In this regard, $D_{e}$ values obtained from the impedance model given in [24] are one order of magnitude higher than $D_{e}$ values extracted from RDEV.

Table 4: Standard Electrochemical Rate Constant and Diffusion Coefficient Values for Deactivated Poap Films Extracted from the Impedance Model Give in [24].

The loss of electroactivity of a polymer film under continuous potential cycling has been attributed to an excessive uptake of doping sites by dopant anions from the external electrolyte solution. Then, after a great number of potential cycles, anions may associate strongly with positive sites of the polymer chains resulting in electrostatic cross-linking, which in turn leads to the polymer deactivation $[29,30]$. However, the prolonged potential 
cycling can also alter the molecular structure of a POAP film, and, hence, its physical properties. Thus, parameters obtained in this work from impedance models strictly represent fitting parameters of the model rather than real physical properties of the polymer. Although one cannot expect a complete coincidence of the electrochemical properties of the polymer film with those predicted by the theory, a qualitative agreement might be expected, especially in order to compare the behaviours of films before and after they have been subjected to an extensive potentiodynamic cycling.

\section{Conclusions}

In this work it is demonstrated that poly(o-aminophenol) films maintain their conducting properties unaltered for about 500 potential cycles within the potential range $-0.2 \mathrm{~V}<E<0.5 \mathrm{~V}$ (SCE) at a scan rate of $0.010 \mathrm{Vs}^{-1}$. However, a loss of conductivity was observed as the number of potential cycles was extended beyond 500. The loss of conductivity of POAP with prolonged potential cycling is demonstrated by the attenuation of the voltammetric response and the decrease of some charge-transport and chargetransfer parameters, such as, the electron diffusion coefficient and the standard electrochemical rate constant obtained from RDEV and EIS measurements. An efficiency of POAP to act as a mediator in the HQ/Q redox reaction was defined in this work. It was found that the efficiency decreases from 100 per cent to 40 per cent as the number of potential cycles increases from about 500 to 5000 .

\section{Acknowledgments}

The author gratefully acknowledges the Consejo Nacional de Investigaciones Científicas y Técnicas (CONICET) and also the Facultad de Ciencias Exactas, National University of La Plata (UNLP).

\section{References}

[1] C. Barbero, J. J. Silber, and L. Sereno, "Electrochemical properties of poly- ortho-aminophenol modified electrodes in aqueous acid solutions," Journal of Electroanalytical Chemistry and Interfacial Electrochemistry, vol. 291, no. 1, pp. 81-101, 1990.

[2] C. Barbero, J. J. Silber, and L. Sereno, "Formation of a novel electroactive film by electropolymerization of orthoaminophenol: Study of its chemical structure and formation mechanism. electropolymerization of analogous compounds," Journal of electroanalytical chemistry and interfacial electrochemistry, vol. 263, no. 2, pp. 333-352, 1989.

[3] T. Ohsaka, S. Kunimura, and N. Oyama, "Electrode kinetics of poly ( o-aminophenol) film prepared by electro-oxidative polymerization of o-aminophenol and its electrochromic properties," Electrochimica acta, vol. 33, no. 5, pp. 639645, 1988.

[4] R. Tucceri, P. Arnal, and A. Scian, "Poly (o-aminophenol) film electrodes: synthesis and characterization and forma- tion mechanisms - a review article," Canadian Journal of Chemistry, vol. 91, no. 2, pp. 91-112, 2012.

[5] D. Scolari and R. Tucceri, "Some applications of nonconducting poly (o-aminophenol) films in bioelectrochemistry and electrocatalysis," Micro and Nanosystems, vol. 3, no. 2, pp. 115-130, 2011.

[6] R. I. Tucceri, C. Barbero, J. J. Silber, L. Sereno, and D. Posadas, "Spectroelectrochemical study of poly-oaminophenol," Electrochimica acta, vol. 42, no. 6, pp. 919927, 1997.

[7] O. Levin, V. Kondratiev, and V. Malev, "Charge transfer processes at poly-o-phenylenediamine and poly-o-aminophenol films," Electrochimica acta, vol. 50, no. 7, pp. 1573-1585, 2005.

[8] M. Miras, A. Badano, M. Bruno, and C. Barbero, "Nitric oxide electrochemical sensors based on hybrid films of conducting polymers and metal phtalocyanines," Portugaliae Electrochimica Acta, vol. 21, no. 3, pp. 235-243, 2003.

[9] J. Yano, H. Kawakami, S. Yamasaki, and Y. Kanno, "Cation capturing ability and the potential response of a poly (oaminophenol) film electrode to dissolved ferric ions," Journal of the Electrochemical Society, vol. 148, no. 2, pp. E61E65, 2001.

[10] M. Lobo, A. Miranda, J. López-Fonseca, and P. Tunon, "Electrocatalytic detection of nicotinamide coenzymes by poly (o-aminophenol)-and poly (o-phenylenediamine)modified carbon paste electrodes," Analytica chimica acta, vol. 325, no. 1, pp. 33-42, 1996.

[11] A. Zhang, C. Cui, and J. Y. Lee, "Metalpolymer interactions in the ag+poly-o-aminophenol system," Journal of Electroanalytical Chemistry, vol. 413, no. 1, pp. 143-151, 1996.

[12] K. L. Chopra and I. Kaur, Thin film phenomena, vol. 137. McGraw-Hill New York, 1969.

[13] R. Tucceri, "A review about the surface resistance technique in electrochemistry," Surface Science Reports, vol. 56, no. 3, pp. 85-157, 2004.

[14] R. Tucceri and D. Posadas, "A surface conductance study of the anion adsorption on gold," Journal of electroanalytical chemistry and interfacial electrochemistry, vol. 191, no. 2, pp. 387-399, 1985.

[15] R. Tucceri and D. Posadas, "Resistive behavior of thin gold film electrodes under direct current polarization," Journal of the Electrochemical Society, vol. 130, no. 1, pp. 104-107, 1983.

[16] C. Barbero, J. Zerbino, L. Sereno, and D. Posadas, “Optical properties of electropolymerized orthoaminophenol," Electrochimica acta, vol. 32, no. 4, pp. 693-697, 1987.

[17] C. Andrieux and J. Saveant, "Electron transfer through redox polymer films," Journal of Electroanalytical Chemistry and Interfacial Electrochemistry, vol. 111, no. 2, pp. 377-381, 1980. 
[18] E. Laviron, "A multilayer model for the study of space distributed redox modified electrodes: Part i. description and discussion of the model," Journal of Electroanalytical Chemistry and Interfacial Electrochemistry, vol. 112, no. 1, pp. 1-9, 1980.

[19] C. Deslouis and B. Tribollet, "Flow modulation techniques in electrochemistry," Advances in Electrochemical Science and Engineering, vol. 2, p. 205, 1992.

[20] A. Bonfranceschi, A. Pérez Córdoba, S. Keunchkarian, S. Zapata, and R. Tucceri, "Transport across poly (oaminophenol) modified electrodes in contact with media containing redox active couples. a study using rotating disc electrode voltammetry," Journal of Electroanalytical Chemistry, vol. 477, no. 1, pp. 1-13, 1999.

[21] C. Barbero, R. Tucceri, D. Posadas, J. Silber, and L. Sereno, "Impedance characteristics of poly-o-aminophenol electrodes," Electrochimica acta, vol. 40, no. 8, pp. 1037-1040, 1995.

[22] C. E. Chidsey and R. W. Murray, "Redox capacity and direct current electron conductivity in electroactive materials," The Journal of Physical Chemistry, vol. 90, no. 7, pp. 14791484, 1986.

[23] W. John Albery, M. G. Boutelle, and A. R. Hillman, "The mechanism of faradaic reactions at the thionine coated electrode," Journal of Electroanalytical Chemistry and Interfacial Electrochemistry, vol. 182, no. 1, pp. 99-111, 1985.

[24] F. J. Nieto and R. Tucceri, "The effect of ph on the charge transport at redox polymer-modified electrodes: an ac impedance study applied to poly ( o-aminophenol) film elec- trodes," Journal of Electroanalytical Chemistry, vol. 416, no. 1, pp. 1-24, 1996.

[25] P. Agarwal, M. E. Orazem, and L. H. Garcia-Rubio, "Measurement models for electrochemical impedance spectroscopy i. demonstration of applicability," Journal of the Electrochemical Society, vol. 139, no. 7, pp. 1917-1927, 1992.

[26] P. Agarwal, O. D. Crisalle, M. E. Orazem, and L. H. GarciaRubio, "Application of measurement models to impedance spectroscopy ii. determination of the stochastic contribution to the error structure," Journal of the Electrochemical Society, vol. 142, no. 12, pp. 4149-4158, 1995.

[27] P. Agarwal, M. E. Orazem, and L. H. Garcia-Rubio, "Application of measurement models to impedance spectroscopy iii. evaluation of consistency with the kramers-kronig relations," Journal of the Electrochemical Society, vol. 142, no. 12, pp. 4159-4168, 1995.

[28] M. E. Orazem, "A systematic approach toward error structure identification for impedance spectroscopy," Journal of Electroanalytical Chemistry, vol. 572, no. 2, pp. 317-327, 2004.

[29] P. Novák, K. Müller, K. Santhanam, and O. Haas, "Electrochemically active polymers for rechargeable batteries," Chemical Reviews, vol. 97, no. 1, pp. 207-282, 1997.

[30] Z. Cai and C. R. Martin, "Electrochemical investigations of electronically conductive polymers: Part vi. mechanism of the redox reactions for the electronically conductive form of polypyrrol," Journal of electroanalytical chemistry and interfacial electrochemistry, vol. 300, no. 1, pp. 35-50, 1991. 